\title{
Human and animal perceptual learning: Some common and some unique features
}

\author{
Chris J. Mitchell \\ University of New South Wales, Sydney, Australia
}

\begin{abstract}
A selective summary of the four contributions to this special issue of Learning \& Behavior on perceptual learning is presented. Mackintosh and Hall propose an associative analysis of perceptual learning. It is argued that Tsushima and Watanabe's psychophysical evidence and Fiser's Bayesian-modeling work represent (in different ways) challenges to the associative approach. Some tentative suggestions are explored with regard to how animal learning theorists might meet these challenges. Finally, the role of awareness in perceptual learning is briefly examined.
\end{abstract}

The four articles in this special issue of Learning \& Behavior provide a diverse range of perspectives on the psychological mechanisms underlying perceptual learning. Two of the authors, Mackintosh and Hall, are associative learning theorists, and their work will be familiar to readers of Learning \& Behavior. Many readers will be sympathetic to the general view expressed by Hall and Mackintosh, that, in principle, the phenomena of perceptual learning are amenable to explanation in associative terms. The work described in the two remaining contributionsone by Fiser and one by Tsushima and Watanabe - might be less familiar to this readership. The present summary and synthesis will attempt to bring together some of the ideas presented in the four preceding papers, with an emphasis on the implications of Tsushima and Watanabe's psychophysical research and Fiser's Bayesian modeling for the associative analysis of perceptual learning.

It is interesting to see how the four contributors define the term perceptual learning. Mackintosh (2009) quotes Fahle's (2002, p. ix) definition, according to which perceptual learning "improves discrimination between stimuli that could not be discriminated before learning." Hall's (2009) definition of perceptual learning (originally stated in Hall, 2008) is very similar to Mackintosh's, but also hints at a mechanism by which improved discrimination might come about. Hall says that perceptual learning is "the learning process (or processes) that increases the effectiveness of the unique stimulus elements and/or reduces that of common stimulus elements, thus facilitating discrimination between similar stimuli" (p. 135). The focus in both of these definitions is on learned differences between initially very similar stimuli.

Tsushima and Watanabe (2009) define perceptual learning as an "improvement on a perceptual or sensory task by practice or experience" (p. 126). Fiser (2009) states that the problem of perceptual learning is to understand "how humans and animals extract novel visual descriptions, features, or chunks from their visual environment and represent them" (p. 142). These definitions of perceptual learning seem much broader than those of Hall (2009) and Mackintosh (2009). Because the main aim of the present article is to examine how the work of Fiser and of Tsushima and Watanabe might inform an associative analysis of perceptual learning, the narrower definition will be adopted. Thus, the focus here will be on learned changes in the discriminability of similar stimuli.

I shall briefly describe the associative approach to perceptual learning, as outlined in the articles by Hall (2009) and Mackintosh (2009), focusing on the finding that nonreinforced exposure to two similar stimuli can enhance their distinctiveness. The results described by Tsushima and Watanabe (2009) and some results from my own laboratory appear to be quite inconsistent with some aspects of the associative analysis. These inconsistencies will be described, and two possible resolutions will be suggested. Then this overview will evaluate Fiser's (2009) suggestion that a Bayesian approach provides a better account of learning than does associative link formation. Before concluding, a very brief comment will be made on Fiser's and Tsushima and Watanabe's claims to have observed perceptual learning outside of awareness.

\section{The Associative Analysis of Perceptual Learning}

Associative learning theorists usually take an elemental approach to perceptual learning. Thus, two similar stimuli are referred to as $\mathrm{AX}$ and $\mathrm{BX} ; \mathrm{X}$ is the common element, and $\mathrm{A}$ and $\mathrm{B}$ are unique elements. In a typical experiment studying perceptual learning following mere exposure (e.g., Mackintosh, Kaye, \& Bennett, 1991), the stimuli might be salty lemon (AX) and sweet lemon (BX). Here, the common element $\mathrm{X}$ is the lemon flavor; the unique elements are the salty and sweet flavors A and B, respectively. 
One group of rats (the preexposed group) is given the opportunity to sample salty and sweet lemon on a number of trials prior to training. The control group is given water in the preexposure phase.

In the subsequent training phase, all rats consume one flavor (e.g., AX) and are then injected with lithium chloride to induce nausea. This training will produce an aversion to AX that may generalize to the similar BX flavor. The common finding is that rats given preexposure to $\mathrm{AX}$ and $\mathrm{BX}$ will consume more $\mathrm{BX}$ on test than will the control rats. That is, the aversion to $\mathrm{AX}$ does not generalize very well to BX in the preexposed rats. This finding is consistent with the idea that rats preexposed to $\mathrm{AX}$ and $\mathrm{BX}$ are better able to tell $\mathrm{AX}$ and $\mathrm{BX}$ apart - a perceptual learning effect.

On the basis of well-established principles of associative learning, Mackintosh (2009) proposes a straightforward mechanism to account for this finding (see also McLaren, Kaye, \& Mackintosh, 1989). He says that, as a consequence of preexposure, $\mathrm{AX}$ and $\mathrm{BX}$ will become latently inhibited. That is, any subsequent conditioning with these stimuli will be retarded. It is important to note that the common element $\mathrm{X}$ receives twice as much exposure as either A or B because it is present on both AX and $\mathrm{BX}$ preexposure trials. Therefore, $\mathrm{X}$ will suffer twice as much latent inhibition as either A or B. In the subsequent training phase, when $\mathrm{AX}$ is followed by illness, the relatively novel A element will overshadow the more familiar $\mathrm{X}$ element, and $\mathrm{X}$ will gain little associative strength. It is, of course, the associative strength of $X$ that determines the degree of generalization of the aversion from AX to BX. Therefore, perceptual learning can be explained by the relative familiarity of common and unique elements and by the resulting latent inhibition suffered by those elements. More generally, this theory suggests that, as two similar stimuli become more familiar, their unique features become relatively more salient. An increase in the salience of the unique features relative to the common features is all that is required to improve the discriminability of AX from BX (see also Hall, 2009).

Mackintosh et al. (1991) provided experimental confirmation that greater exposure to the common element than to the unique elements of two similar stimuli can produce a perceptual learning effect. They presented one group of rats with just the $\mathrm{X}$ element in preexposure. This preexposure reduced the degree to which an aversion to AX generalized to BX on test. We have since observed a similar effect in our laboratory (Wang \& Mitchell, 2009), using human participants and the colored checkerboard stimuli presented as Figure 7 in Hall (2009, p. 139 in this issue). We found that repeated exposures to the common $\mathrm{X}$ feature enhanced discrimination of $\mathrm{AX}$ from $\mathrm{BX}$ when the stimuli were tested in a same-different task.

Another phenomenon that should briefly be mentioned is the intermixed-blocked effect (Honey, Bateson, \& Horn, 1994). It is now well established, in a range of species and procedures (see, e.g., Blair \& Hall, 2003; Lavis \& Mitchell, 2006; Mitchell, Kadib, Nash, Lavis, \& Hall, 2008; Mitchell, Nash, \& Hall, 2008; Mundy, Honey, \& Dwyer, 2007; Symonds \& Hall, 1995), that intermixed presentations of $\mathrm{AX}$ and $\mathrm{BX}(\mathrm{AX} \mathrm{BX} \mathrm{AX} \mathrm{BX} \ldots$...) produce better perceptual learning than do blocked presentations of the same stimuli (AX AX AX ... BX BX BX ...). The intermixed-blocked effect has received a great deal of attention recently because it cannot be explained in terms of the relative exposure to (and therefore familiarity of) the common and unique features; this is equivalent in the intermixed and blocked conditions. Nevertheless, three of the four theories that have been proposed to account for the intermixed-blocked effect (see Mackintosh, 2009)_ and much of the data (e.g., Blair \& Hall, 2003; Mitchell, Kadib, et al., 2008) — suggest that the unique features $\mathrm{A}$ and $\mathrm{B}$ are more salient following intermixed than following blocked preexposure (see Hall, 2009).

\section{The Challenge From Psychophysics}

Some experiments conducted by Watanabe, Náñez, and Sasaki (2001) appear to challenge one of the major assumptions underlying the associative analysis of perceptual learning (and, indeed, associative learning) - that novel stimuli are more salient than familiar stimuli. The stimulus arrays presented to participants in Watanabe et al.'s experiments contained many dots moving in different directions. Some percentage of those dots moved coherently in the same direction, and the remainder moved at random. When $10 \%$ of those dots moved in the same direction, participants often reported coherent motion in that direction. However, when only $5 \%$ of the dots moved in the same direction, participants did not perceive coherent motion.

Watanabe et al.'s (2001) experiments showed that the skill in detecting coherent motion could be improved. Participants were given many days of exposure to arrays in which $5 \%$ of the dots moved in the same direction (e.g., toward the top of the screen). As a consequence, participants were given much more exposure to dots moving in one direction than to those moving in any other direction, but they were not aware of this manipulation. In fact, they did not perceive coherent motion in the $5 \%$ displays, even after training. On test, participants were presented with $10 \%$ coherent motion displays, which they were able to detect reasonably well, in a range of directions. Detection of motion in the preexposed direction (e.g., toward the top of the screen) was significantly better than that for any other direction. That is, the familiar stimulus (direction of movement) was higher in salience than were the less familiar stimuli.

We have recently found a similar effect in humans, using our checkerboard stimuli (Lavis, Kadib, Hall, \& Mitchell, 2009). Participants were exposed to six stimuli, all intermixed with each other. These stimuli were the compounds $\mathrm{AX}, \mathrm{BX}, \mathrm{CX}$, and $\mathrm{DX}$, as well as the elements $\mathrm{A}$ and $\mathrm{B}$ alone. On test, discrimination of $\mathrm{AX}$ and $\mathrm{BX}$ on a same-different task was better than that of $\mathrm{CX}$ and DX. That is, additional exposures to the unique features produced better discrimination. In a related experiment, we investigated recognition of unique features following intermixed and blocked preexposure. On the basis of the findings above, we postulated that intermixed preexposure to $\mathrm{AX}$ and $\mathrm{BX}$ might improve discrimination 
relative to blocked preexposure because it allows better encoding of A and B in memory. This idea was supported; when the unique stimulus features were presented alone in a recognition-test task, those features that were preexposed on the intermixed schedule were recognized better than those preexposed on the blocked schedule (Lavis et al., 2009). If, as suggested by Hall (2009), intermixed preexposure increases the salience or effectiveness of the unique features, ${ }^{1}$ then, again, it would appear that it is the features that are better encoded in memory that are highest in salience.

These experiments imply that there is a limit to the common ground shared by humans and animals in the way that perceptual learning takes place. On the positive side, preexposure to the common $\mathrm{X}$ features of two similar stimuli, AX and BX, has been shown to promote perceptual learning in both rats (Mackintosh et al., 1991) and humans (Wang \& Mitchell, 2009; Wills \& McLaren, 1998). Further evidence for a common mechanism comes from the intermixed-blocked effect, which has been observed in chicks (see, e.g., Honey et al., 1994) and rats (see, e.g., Symonds \& Hall, 1995), as well as in humans (see, e.g., Lavis \& Mitchell, 2006; Mundy et al., 2007). There is also, as Hall (2009) points out, abundant evidence that explicit training, such as differential reinforcement, can produce similar perceptual learning effects in humans and animals.

However, the finding that preexposure to the unique features alone enhances discrimination in human perceptual learning studies (Lavis et al., 2009; Watanabe et al., 2001) cannot be accounted for in any straightforward way by associative theory. It is quite inconsistent with Mackintosh's (2009) idea that perceptual learning is partly due to the greater exposure given to the common than to the unique stimulus features. Furthermore, this effect is not seen in animals (Mackintosh et al., 1991). Taken together, the data suggest that similar stimuli AX and BX are most easily discriminated by humans when all of the features (A, B, and $\mathrm{X}$ ) are well encoded in memory. However, discrimination performance in animals seems to be best, all other things being equal, when the unique features are less familiar (and, on this view, therefore, more salient) than the common features. In the section that follows, two accounts will be given for this discrepancy between the behavior of humans and animals in perceptual learning experiments. The first suggests that the difference lies in the learning process. The second suggests that the way in which the tests are conducted is important.

\section{Self-Supervised Learning in Humans but Not in Animals}

One explanation for differences observed between some human and animal studies is suggested by Mackintosh (2009). He argues for an important distinction between supervised and unsupervised learning. As he points out, if reinforcement is given for responses to AX but not for those in BX (supervised learning), then any improvement in discrimination of $\mathrm{AX}$ and $\mathrm{BX}$ on test could be explained quite easily as the consequence of associative mechanisms (see also Hall, 2009). It is perceptual learning following mere exposure (unsupervised learning) that requires a more sophisticated theoretical analysis. Mackintosh further argues that, even when no explicit reinforcement is given, human perceptual learning tasks are very often cases of supervised learning. As he points out, participants will often assume (and are sometimes instructed) that their task is to look for differences in the similar stimuli presented in the preexposure phase. What else could the experiment be about? The result of this active search is that when participants detect differences, they receive reinforcement because they have succeeded in their task. This reinforcement may serve to maintain attention, in a top-down manner, to the differences between the stimuli. Mackintosh refers to this as selfsupervised learning.

Self-supervised learning offers an explanation for why humans are better at discriminating AX and BX when $\mathrm{A}$ and $\mathrm{B}$ are familiar, whereas animals are better at discriminating $\mathrm{AX}$ and $\mathrm{BX}$ when $\mathrm{A}$ and $\mathrm{B}$ are relatively novel. A human participant's goal of maintaining attention to A and B may be more easily achieved when A and B are more familiar. This may be because a familiar feature, one for which a stronger representation exists in memory, is more easily detected. Additional exposure to the unique features will, of course, facilitate this process. In contrast, when animals are given nonreinforced preexposure, it could be argued to be true unsupervised learning, because the animals do not know and are unlikely to guess what their task is. If the learning in animals is unsupervised, and if there is no deliberate search for the distinguishing features of the two stimuli, then the consequence of additional exposures to $\mathrm{A}$ and $\mathrm{B}$ will be to reduce the salience of those features.

Watanabe et al.'s (2001) results, at least, do not seem to be explicable in terms of self-supervised learning: If participants are not aware of the stimuli during preexposure, then the learning that takes place is almost certainly unsupervised. To examine whether Watanabe et al.'s findings can be generalized to other stimuli, it would be interesting to see whether additional exposures to the unique features $\mathrm{A}$ and $\mathrm{B}$ can improve perceptual discrimination of other complex stimuli, such as our checkerboards, in a truly unsupervised learning procedure. It might not, however, be possible to produce learning outside of awareness with these stimuli.

Another possibility would be to use the approach taken by McLaren, Leevers, and Mackintosh (1994) and Goldstone (1994), in which participants are required to categorize AX and BX together. Here, $\mathrm{X}$ is the feature that is relevant to the categorization and should therefore be the focus of the participants' attention. In the experiments of McLaren et al. and Goldstone, discrimination of AX and $\mathrm{BX}$ improved on test as a consequence of categorization training. Additional exposures to A and B (in such a way that participants are not alerted to the true nature of the task) may further improve discrimination performance on test. Such a result would be very difficult to explain in terms of self-supervision; any supervised learning that occurs would be expected to increase attention to $\mathrm{X}$, the common feature that allows successful performance on the categorization task. 
If discrimination in these future studies of unsupervised learning in humans improves as a consequence of additional exposures to the unique features, then how could the discrepancy between human and animal learning be explained? One possibility lies in the type of test used to measure discriminability in humans and animals.

\section{Differences in Testing Procedures Used in Animals and Humans}

When animal researchers test stimulus discriminability, they condition a new response to stimulus AX (e.g., a taste aversion) and then observe the degree of generalization of this response to BX. Researchers testing human participants are able to take a more direct approach: They can simply ask participants whether the two stimuli presented are the same or different. These two tasks may be sensitive to two quite different consequences of stimulus preexposure.

First, when two similar stimuli are presented, the animal is given the opportunity to detect the various stimulus features, both common and unique, and to encode those features in memory. Once the stimulus features are well encoded in memory, they may be more easily identified when presented on test. The second thing that the animal learns is that the various features presented do not predict or signal anything of significance. Thus, the features become latently inhibited. As Mackintosh (2009) points out, the common features will suffer more latent inhibition than will the unique features because the common features are presented twice as often.

Latent inhibition can be expected to have a profound effect on the test of perceptual learning usually used in animal conditioning studies. If $\mathrm{X}$ is low in associability relative to $\mathrm{A}$ (due to latent inhibition), then $\mathrm{A}$ will gain the majority of the available associative strength when $\mathrm{AX}$ is conditioned. Generalization of the conditioned response (CR) from $\mathrm{AX}$ to $\mathrm{BX}$ will, therefore, be weak. In other words, AX and BX will appear to be easily discriminated. If additional exposures to $\mathrm{A}$ and $\mathrm{B}$ are given, the associability of these features will decline. As a consequence, the common $\mathrm{X}$ feature will compete more readily for associative strength on AX conditioning trials, and stronger generalization will be observed between AX and BX. This will be interpreted as a reduction in discriminability.

In a same-different task, no conditioning takes place. It is unlikely that the associability of $\mathrm{A}, \mathrm{B}$, and $\mathrm{X}$ and any latent inhibition they may have suffered will affect performance on this task. Performance may be determined by the ease with which the various stimulus elements - in particular, the unique features - can be identified (Gibson, 1969). If so, performance will be expected to be improved by additional exposures to the unique features. Therefore, the differences observed between human and animal studies could be due to the different test tasks used.

The way to test this idea would be to present animals with $\mathrm{AX}$ and $\mathrm{BX}$ in a same-different task following preexposure (e.g., Brooks \& Wasserman, 2008). The straightforward prediction is that additional exposures to A and B will improve performance on this task. It may appear at first that such a finding could be explained in terms of self-supervision. A same-different task requires subjects to look for a difference between the stimuli; this is what allows the A and B features to increase in salience on test. But it is important to be clear that this top-down mechanism can only maintain attention to those features already detected. It does not provide a mechanism by which features are detected (and therefore encoded in memory) in the first instance. Furthermore, in this proposed experiment, the same-different task is implemented on test, so self-supervision cannot explain any differences observed as a consequence of experimental manipulations implemented in the preexposure phase.

An alternative approach would be to give human participants conditioning with $\mathrm{AX}$ as the conditioned stimulus (CS). Generalization to BX may show the same pattern as is observed in animals. However, it may be difficult to obtain this effect; latent inhibition is a fragile effect in humans.

In summary, when humans are learning to discriminate between AX and BX, they appear to benefit from additional exposures to $\mathrm{A}$ and $\mathrm{B}$. This effect is similar to the easy-hard effect described by Mackintosh (see also, e.g., Scahill \& Mackintosh, 2004). Animals do not show this effect unless reinforcement is given in the preexposure phase. One explanation for the result obtained in humans, therefore, is that humans receive reinforcement in preexposure. That is, self-supervised learning occurs in humans, which serves to reinforce detection of the unique features, even when no explicit reinforcement is given. However, in Watanabe et al.'s (2001) procedure, participants are not aware of the stimuli in preexposure. Even under these circumstances, participants' discrimination performance appears to benefit from exposures to the unique cue. Perhaps, therefore, additional exposures to the unique features $\mathrm{A}$ and $\mathrm{B}$ increase generalization of a CR from AX to BX (the test task usually used in animal studies), even though the perceptual discriminability of these two stimuli (as measured in a same-different task) is enhanced.

One very general implication of this analysis that may seem self-evident to many is that generalization of a $\mathrm{CR}$ from one stimulus to another may not be a good indicator of the perceptual discriminability of the two stimuli. This conclusion will, perhaps, be more important to psychophysicists than to associative learning theorists. As Mackintosh (2009) points out, Fahle's (2002) definition of perceptual learning requires that a change in perception take place. Mackintosh explicitly disagrees with this position and would like to include a broader range of sometimes-nonperceptual phenomena within the definition. This disagreement may limit the extent to which the findings of researchers from different theoretical traditions can be integrated; to some extent, animal learning researchers and psychophysicists may simply be investigating different phenomena. Nevertheless, as Hall (2009) points out, the similarities between human and animal perceptual learning are impressive and suggest that much closer integration between the two traditions is possible. 


\section{The Bayesian Approach to Learning}

Fiser's contribution to this special issue addresses the way in which we construct mental representations of complex visual images. His approach departs from the elemental analysis of stimulus representation that is the starting point for the associative analysis of perceptual learning. In Fiser's experiments, combinations of shapes in a particular configuration serve as stimuli (e.g., Fiser \& Aslin, 2001, 2002). The task for the participant is to ignore coincidental shape pairings and learn which shapes regularly go together to form a stimulus. Fiser (2009) describes many experiments of this kind, which test a range of different conditions. For example, in one such experiment, participants were presented with four-shape stimuli (combos) in training and were then asked whether they recognized those stimuli, or parts of those stimuli (e.g., a pair of shapes contained within the combo), on test. Fiser demonstrates an impressive fit between his computational model, developed within a Bayesian framework, and the human recognition data observed in his experiments.

On the face of it, Fiser's (2009) task seems more like a standard associative-learning task than a perceptuallearning task. Thus, participants must learn which shapes go together; they are not required to discriminate very similar stimuli (or combinations). We would still want to argue that this is a form of perceptual learning. It seems reasonable to suppose that if one has two separate and strong representations of two similar stimuli in memory, then discrimination of those two stimuli will be enhanced. In fact, McLaren and Mackintosh (2000) provide one mechanism by which associations between stimulus elements - unitization - might produce better discrimination. If we allow that Fiser's task requires a form of perceptual learning, the question then is whether Bayesian-model averaging provides a better account of this kind of learning than do traditional associative models. Fiser also makes the more general point that Bayesian models may provide a better explanation of all associative-learning phenomena than do associative (link-based) learning models.

When comparing the Bayesian and associative approaches, it is important to be clear that these are both frameworks. The Bayesian framework assumes that learning is a kind of statistical inference. The associative framework assumes that learning results from the linking of representational nodes, so that the presentation of one stimulus is able to activate the representation of another. Within each framework, a great many theories are possible. Consequently, finding that a single specific Bayesian model outperforms a single specific associative model does not suggest that the Bayesian framework as a whole is superior to the associative framework, or vice versa. The great variety of link-based models that have been proposed allows the associative-learning theorist great flexibility for explaining a given data set. Proponents of the Bayesian approach are generally sensitive to this issue and test their models against a variety of competing models.

In his own work, Fiser compares a Bayesian model, the "ideal learner," with four other models and finds that the ideal learner provides the best fit to the human behavioral data. The four models against which the ideal learner is compared include a very simple frequency-counting model (Type 1); a model that counts pairwise co-occurrences of shapes (Type 2); a model that calculates the conditional probability that two shapes will co-occur (Type 3); and, finally, a more complex model (Type 4) described by Fiser (citing Dayan \& Abbott, 2001) as an implementation of the most studied associative model of unsupervised learning in the literature.

Despite the range of models tested, many readers may feel that Fiser (2009) does not provide a thorough enough test of associative theory. They may argue that some other associative model would provide a more accurate fit to the observed data than that provided by the ideal learner. Probably the most obvious candidate theory is the influential model of perceptual learning proposed by McLaren and Mackintosh (2000). These authors proposed a variety of mechanisms that, taken together, might provide an account of Fiser's results and suggest ways in which the theories could be further tested. However, of course, it is for the proponents of the associative approach to conduct this research and to meet the challenges posed by Fiser's very interesting findings. Conversely, there are findings from the animal literature, such as the intermixed-blocked effect, that are not accounted for by the ideal learner. Thus, the Bayesian approach also requires further refinement to accommodate all of the available data.

Fiser (2009) describes some of the Bayesian theories as "a statistical reformulation of different learning theories" (p. 141). One possible outcome of future theoretical development and refinement, therefore, is that associative models can, at least to some extent, be reformulated as Bayesian inference, and vice versa. If this is the case, then the approach of testing individual Bayesian models against individual associative models would be of limited value. ${ }^{2}$ There is, though, one fundamental difference between the Bayesian and associative approaches that can be tested. From the Bayesian perspective, "the goal of learning is to develop a representation of the outside world including the relations between the CS and the US [unconditioned stimulus]" (Fiser, 2009, p. 149). In contrast, the traditional associative view is that learning involves the formation of a link between the nodes that represent the relevant stimuli, such as a CS and US. The link itself is not a representation; it is simply a psychological mechanism that allows activation to be transmitted from one node to another. At least in humans, the available evidence seems to favor the Bayesian approach: Learning does appear to involve the acquisition of a belief about relationships between events in the world (see Mitchell, De Houwer, \& Lovibond, in press). An interesting possibility, however, is that perceptual learning will turn out to be quite different. When we perceive an object differently as a consequence of exposure, it may be because links form between the elements of that object, allowing one element to activate the other.

\section{Perceptual Learning Outside of Awareness}

Both Fiser (2009) and Tsushima and Watanabe (2009) suggest that perceptual learning may take place outside 
of awareness. Animal learning theorists are usually interested in this issue only to the extent that it might reveal the operation of "true" associative processes in humans or prevent self-supervised learning from taking place. However, this issue is of great interest to many human learning theorists. Past demonstrations of learning outside of awareness have, since their publication, very often been shown to be open to an alternative interpretation (e.g., Lovibond \& Shanks, 2002; Shanks \& St. John, 1994). But Watanabe et al.'s (2001) results, in particular, are very persuasive. Their participants appear to have been unaware of the presence of the 5\% coherent motion they were exposed to, but they learned nevertheless. Furthermore, when $10 \%$ coherent motion (that could be detected by participants) was used in the preexposure phase, no perceptual learning was observed (Tsushima, Seitz, \& Watanabe, 2008). Because unaware learning is a topic of considerable theoretical interest in its own right, and because the data presented by Watanabe and coworkers are compelling, it is important that these results be subjected to the closest scrutiny.

\section{Conclusions}

The four contributions in this special issue raise a number of points that are fundamental to our understanding of perceptual learning both in humans and in animals. These articles come from three quite unconnected research disciplines. I hope that this overview will, to some extent, help to highlight the ways in which these research areas are (and are not) related. It seems to me that Fiser, Tsushima, and Watanabe were extremely good choices to represent the nonassociative perspectives on perceptual learning. Both of the articles from these contributors make contact with the perceptual-learning work conducted in the associative tradition, and in quite different ways. Perhaps more important, both articles present evidence that challenges existing associative accounts of perceptual learning. Associative learning theorists are, however, famously ingenious, and they undoubtedly will offer a robust response.

\section{AUTHOR NOTE}

Correspondence concerning this article should be addressed to C. J. Mitchell, School of Psychology, University of New South Wales, Sydney, NSW 2052, Australia (e-mail: chris.mitchell@unsw.edu.au).

\section{REFERENCES}

Blair, C. A. J., \& Hall, G. (2003). Perceptual learning in flavor aversion: Evidence for learned changes in stimulus effectiveness. Journal of Experimental Psychology: Animal Behavior Processes, 29, 39-48.

Brooks, D. I., \& Wasserman, E. A. (2008). Same/different discrimination learning with trial-unique stimuli. Psychonomic Bulletin \& Review, 15, 644-650.

Dayan, P., \& Аввотт, L. F. (2001). Theoretical neuroscience: Computational and mathematical modeling of neural systems. Cambridge, MA: MIT Press.

FAHLe, M. (2002). Introduction. In M. Fahle \& T. Poggio (Eds.), Perceptual learning. Cambridge, MA: MIT Press.

FISER, J. (2009). Perceptual learning and representational learning in humans and animals. Learning \& Behavior, 37, 141-153.

FISER, J., \& ASLIN, R. N. (2001). Unsupervised statistical learning of higher-order spatial structures from visual scenes. Psychological Science, 12, 499-504.

FISER, J., \& ASLIN, R. N. (2002). Statistical learning of higher-order temporal structure from visual shape sequences. Journal of Experimental Psychology: Learning, Memory, \& Cognition, 28, 458-467.

Gibson, E. J. (1969). Principles of perceptual learning and development. Cambridge, MA: MIT Press.

Goldstone, R. L. (1994). Influences of categorization on perceptual discrimination. Journal of Experimental Psychology: General, 123, 178-200.

Hall, G. (2008). Perceptual learning. In J. Byrne (Editor-in-Chief) \& R. Menzel (Vol. Ed.), Learing and memory: A comprehensive reference. Vol. 1: Learning theory and behavior (pp. 103-121). Amsterdam: Elsevier.

HALL, G. (2009). Perceptual learning in human and nonhuman animals: A search for common ground. Learning \& Behavior, 37, 133-140.

Honey, R. C., Bateson, P., \& Horn, G. (1994). The role of stimulus comparison in perceptual learning: An investigation with the domestic chick. Quarterly Journal of Experimental Psychology, 47B, 83-103.

Lavis, Y., Kadib, R., Hall, G., \& Mitchell, C. J. (2009). Memory for, and salience of, the unique features of similar stimuli in perceptual learning. Manuscript in preparation.

LAVIS, Y., \& MitcheLL, C. (2006). Effects of preexposure on stimulus discrimination: An investigation of the mechanisms responsible for human perceptual learning. Quarterly Journal of Experimental Psychology, 59, 2083-2101.

Lovibond, P. F., \& Shanks, D. R. (2002). The role of awareness in Pavlovian conditioning: Empirical evidence and theoretical implications. Journal of Experimental Psychology: Animal Behavior Processes, 28, 3-26.

Mackintosh, N. J. (2009). Varieties of perceptual learning. Learning \& Behavior, 37, 119-125.

Mackintosh, N. J., Kaye, H., \& Bennett, C. H. (1991). Perceptual learning in flavour aversion conditioning. Quarterly Journal of Experimental Psychology, 43B, 297-322.

Mclaren, I. P. L., Kaye, H., \& Mackintosh, N. J. (1989). An associative theory of the representation of stimuli: Applications to perceptual learning and latent inhibition. In R. G. M. Morris (Ed.), Parallel distributed processing: Implications for psychology and neurobiology (pp. 102-130). Oxford: Oxford University Press.

McLaren, I. P. L., Leevers, H. J., \& Mackintosh, N. J. (1994). Recognition, categorization, and perceptual learning (or, how learning to classify things together helps one tell them apart). In C. Umiltà \& M. Moscovitch (Eds.), Attention and performance XV: Conscious and nonconscious information processing (pp. 889-909). Cambridge, MA: MIT Press.

McLaren, I. P. L., \& Mackintosh, N. I. (2000). An elemental model of associative learning: I. Latent inhibition and perceptual learning. Animal Learning \& Behavior, 28, 211-246.

Mitchell, C. J., De Houwer, J., \& Lovibond, P. F. (in press). The propositional nature of human associative learning. Behavioral \& Brain Sciences.

Mitchell, C. [J.], Kadib, R., Nash, S., Lavis, Y., \& Hall, G. (2008). Analysis of the role of associative inhibition in perceptual learning by means of the same-different task. Journal of Experimental Psychology: Animal Behavior Processes, 34, 475-485.

Mitchell, C. [J.], Nash, S., \& Hall, G. (2008). The intermixedblocked effect in human perceptual learning is not the consequence of trial spacing. Journal of Experimental Psychology: Learning, Memory, \& Cognition, 34, 237-242.

Mundy, M. E., Honey, R. C., \& Dwyer, D. M. (2007). Simultaneous presentation of similar stimuli produces perceptual learning in human picture processing. Journal of Experimental Psychology: Animal Behavior Processes, 33, 124-138.

SCAHILl, V. L., \& MACKInTOSH, N. J. (2004). The easy to hard effect and perceptual learning in flavor aversion conditioning. Journal of Experimental Psychology: Animal Behavior Processes, 30, 96-103.

Shanks, D. R., \& St. John, M. F. (1994). Characteristics of dissociable human learning systems. Behavioral \& Brain Sciences, 17, 367-447.

Symonds, M., \& Hall, G. (1995). Perceptual learning in flavor aversion 
learning: Roles of stimulus comparison and latent inhibition of common stimulus elements. Learning \& Motivation, 26, 203-219.

Tsushima, Y., Seitz, A. R., \& Watanabe, T. (2008). Task-irrelevant learning occurs only when the irrelevant feature is weak. Current Biology, 18, R516-R517.

Tsushima, Y., \& Watanabe, T. (2009). Roles of attention in perceptual learning from perspectives of psychophysics and animal learning. Learning \& Behavior, 37, 126-132.

WANG, S.-L., \& Mitchell, C. J. (2009). Manipulations of the salience of common and unique features in human perceptual learning: Findings from a same-different task and eye gaze. Manuscript in preparation.

Watanabe, T., NáÑez, J. E., \& SASaki, Y. (2001). Perceptual learning without perception. Nature, 413, 844-848.

Wills, A. J., \& McLaren, I. P. L. (1998). Perceptual learning and free classification. Quarterly Journal of Experimental Psychology, 51B 235-270.

\section{NOTES}

1. The only suggested alternative to this view is that the unique features inhibit one another as a consequence of intermixed preexposure (McLaren \& Mackintosh, 2000). However, it is not at all obvious how better recognition performance to $\mathrm{A}$ and $\mathrm{B}$ might be related to inhibition between $\mathrm{A}$ and $\mathrm{B}$.

2. A similar argument is that associative models are at a lower level of explanation than Bayesian models, because it is possible to implement Bayesian models in connectionist networks. However, the associative models tested by Fiser (2009) are psychological models. They are not (like connectionist models) implementations.

(Manuscript received January 28, 2009; accepted for publication January 29, 2009.) 